\title{
3D Geometric and Haptic Modeling of Hand-Woven Textile Artifacts
}

\author{
Hooman Shidanshidi ${ }^{1}$, Fazel Naghdy ${ }^{1}$, Golshah Naghdy $^{1}$, and Diana Wood Conroy ${ }^{2}$ \\ ${ }^{1}$ School of Electrical, Computer and Telecommunications Engineering, Faculty of Informatics, \\ University of Wollongong, Australia \\ ${ }^{2}$ Faculty of Creative Arts, University of Wollongong, Australia \\ hooman@uow.edu.au, fazel@uow.edu.au, golshah@uow.edu.au, \\ dconroy@uow.edu.au
}

\begin{abstract}
Haptic Modeling of textile has attracted significant interest over the last decade. In spite of extensive research, no generic system has been proposed. The previous work mainly assumes that textile has a 2D planar structure. They also require time-consuming objective measurement of textile properties in mechanical/physical model construction. A novel approach for haptic modeling of textile is proposed to overcome the existing shortcomings. The method is generic, assumes a 3D structure textile artifact, and deploys computational intelligence to estimate textile mechanical and physical properties. The approach is designed primarily for display of textile artifacts in museums. The haptic model is constructed by superimposing the mechanical model of textile over its 3D geometrical model. Digital image processing is applied to the still image of textile to identify its pattern and structure. In order to deal with the nonlinearities associated with the textile, a fuzzy rule-based expert system is deployed. This information is then used to generate a 3D geometric model of the artifact in VRML. Selected mechanical and physical properties of the textile are estimated by an artificial neural network with the textile geometric characteristics and yarn properties as inputs. The neural network learning and verification and validation processes are carried out by a sample data set. The mechanical properties are used in the construction of the textile mechanical model. The haptic rendered model is generated by superimposing the physical/mechanical model over the 3D geometric model. This model has been implemented and rendered in Reachin environment, provided an interactive Virtual Reality environment where the user can navigate the graphic 3D presentation of the textile and touch it by a haptic device. Different samples have been modeled and the whole approach has been validated. The interface can be provided in both in the physical environment and through the cyberspace. The validation of method indicates the feasibility of the approach and its superiority to other haptic modeling algorithms.
\end{abstract}

Keywords: Haptic, Fuzzy logic, Neural Network, Hand-woven textile.

\section{Introduction}

Haptic Modeling of textile has attracted significant interest over the last decade. In the work conducted by Govindaraj et al a haptic system for virtual fabric handling 
experience was developed [1]. The approach was limited to static modeling and used Kawabata machine to calculate the mechanical properties of textile. Another important project was HAPTEX $[2,3]$, in which a similar approach for development and validation of haptic model of fabric was developed.

In spite of extensive research, no generic system has yet been proposed. The majority of the haptic models developed in the previous work assume a 2D mesh model for textile which is not an accurate representation of the geometric configuration of the textile. In addition, they are based on empirical parameters obtained from textile samples using specialized instruments such as Kawabata system. The process is often time consuming and elaborate, consisting of manual measurement of physical and mechanical properties of the artifacts. The development of a generic approach for 3D haptic modeling of hand-woven textile artifacts is pursued in this work.

In the proposed approach, the textile pattern and structure are recognized by digital processing of the artifact still image. A fuzzy-rule based expert system is developed to perform the recognition process. The data obtained in this process is employed to automatically generate the 3D geometric model of the artifact in VRML. The mechanical properties of the artifact are estimated by processing the textile geometric characteristics and yarn properties in a neural network system. These mechanical properties are then deployed in the construction of the textile mechanical model. The mechanical model is superimposed over the $3 \mathrm{D}$ geometric model to construct the haptic model. The proposed system is validated using a number of artifact samples.

Overall, the work conduced in this study offers a novel 3D generic haptic modeling for textile artifacts. It can be deployed in museums providing an opportunity for the visitors to touch unique samples of hand-woven textile artifacts. The approach is costeffective, reliable and reproducible, as the haptic modeling of these samples doesn't need time-consuming and costly laboratory conditions.

In the remainder of the paper, the image processing method and the fuzzy rule-base expert system deployed in the construction of the geometric model are described. The outcome is a 3D geometric model of the artifact in VRML that could be explored in a virtual reality world viewer. Similarly, the neural network model designed to estimate the mechanical characteristics of an artifact is presented. The approach is validated and conclusion is made.

\section{Textile Pattern Recognition}

A woven fabric has a complicated structure made of the cross combination of the wrap and weft yarns in a two-dimensional lattice structure. Textile weave pattern recognition has attracted many researchers since the mid-1980's. The problem field could be divided into crossed-points detection that deals with interlacing areas between wrap and weft yarns and crossed-states detection which determines which yarn is over the other in the interlacing areas.

Different approaches have been proposed to deal with both problems. The developed approaches, however, have proved to be inadequate to deal with the non-rigidity of the hand-woven artifacts. In this work, a new algorithm is developed to address such shortcomings [4]. An overview of the approach is provided in this section. 


\subsection{Crossed-Points Detection}

The methods reported in the literature for crossed-points detection can be categorized into two groups:

- Employing Fourier filtering techniques to find periodic weave pattern in a woven fabric image by either identification of the peaks in the power spectrum image [5-7] or finding the peak points of the autocorrelation function of the gray level data in warp and weft directions [8].

- Identifying the peaks in accumulation gray level values in vertical and horizontal directions pixels $[9,10]$.

In this work, the second approach is deployed and a piecewise-linear algorithm is introduced to model the non-linearities in the structure of the fabric. The image features - representing the peaks of local accumulating gray level values for each vertical and horizontal image slice - are extracted. A fuzzy rule-base engine is then applied to process the image features and identify the structure of the artifact.

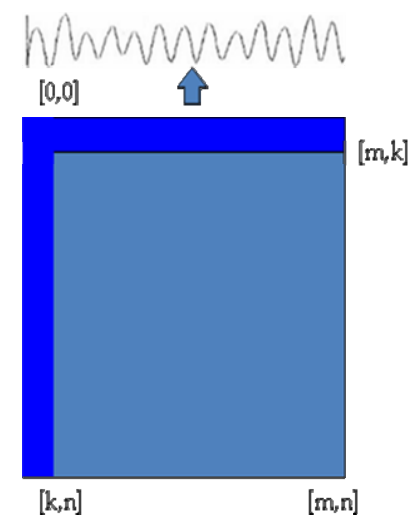

Fig. 1. Image slicing and accumulating gray level curve

The image matrix $\omega_{\mathrm{m}^{*} \mathrm{n}}$ is sliced horizontally and vertically. The image slices $S V_{i}$ in vertical and $S_{i}$ in horizontal directions are defined as elements of $\omega$ matrix which is represented by a ', operator in (1) and (2), where $\mathrm{k}$ is the thickness of the image slice:

$$
\begin{array}{r}
S V_{i}=\omega_{\mathrm{i} * \mathrm{k}, 0} \_\omega_{\mathrm{i} * \mathrm{k}, \mathrm{n}-} \omega_{(\mathrm{i}+1) * \mathrm{k}, 0} \_\omega_{(\mathrm{i}+1) * \mathrm{k}, \mathrm{n}} \\
\text { and } 0 \leq \mathrm{i} \leq\left\lceil\frac{m}{k}\right\rceil-1 \\
S H_{i}=\omega_{0, \mathrm{i} * \mathrm{k} \_} \omega_{\mathrm{m}, \mathrm{i} * \mathrm{k}-} \omega_{0,(\mathrm{i}+1) * \mathrm{k}} \_\omega_{\mathrm{m},(\mathrm{i}+1) * \mathrm{k}} \\
\text { and } 0 \leq \mathrm{i} \leq\left\lceil\frac{n}{k}\right\rceil-1
\end{array}
$$


By considering the $\Omega_{\mathrm{x}, \mathrm{y}}$ the gray level conversion function of the pixel in position $\mathrm{x}$ and $\mathrm{y}, \alpha_{\mathrm{i}}$ a vector with $\mathrm{m}$ elements as the accumulating gray level values for the vertical slice $\mathrm{i}, \mathrm{SV}_{\mathrm{i}}$ could be calculated as:

$$
\alpha_{i, j}=\sum_{z=0}^{k} \Omega\left(S V_{i}^{z, j}\right) \quad \text { for } 1 \leq \mathrm{j} \leq \mathrm{n}
$$

And the same for $\beta_{\mathrm{i}}$ a vector with $\mathrm{n}$ elements as the accumulating gray level values for the horizontal slice $\mathrm{i}, \mathrm{SH}_{\mathrm{i}}$ :

$$
\beta_{i, j}=\sum_{z=0}^{k} \Omega\left(S H_{i}^{j, z}\right) \quad \text { for } 1 \leq \mathrm{j} \leq \mathrm{m}
$$

Finally, Feature point set $\mu_{\mathrm{vi}}$ for the vertical slice $i$ and $\mu_{\mathrm{hi}}$ for the horizontal slice $i$ are calculated as below:

$$
\begin{array}{ll}
\mu_{v i}=\left\{p \mid p \in \min \left(\alpha_{i}\right)\right\} & \text { for } 1 \leq \mathrm{i} \leq \mathrm{m} \\
\mu_{h i}=\left\{p \mid p \in \min \left(\beta_{i}\right)\right\} & \text { for } 1 \leq \mathrm{i} \leq \mathrm{n}
\end{array}
$$

While ideally, the minimum peaks of the autocorrelation show the yarn border in that section, variation in artifact pattern, sometimes results in false peaks. Hence, a special filter is developed to identify real peaks in the yarn boarder. Figure 2 illustrates this filtering mechanism.

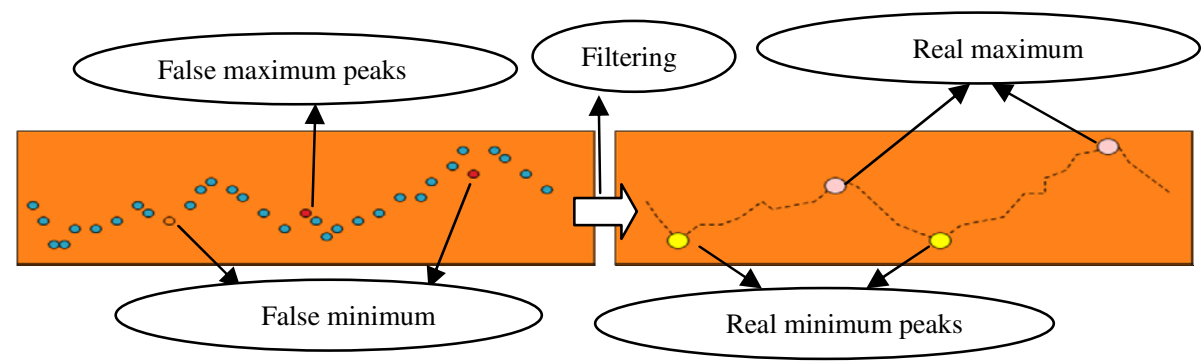

Fig. 2. Intelligent filtering mechanism for removing the false peaks and picking the real peaks

In addition, a mechanism is also required to locate the corresponding points in different slices belonging to one specific yarn boarder. At this stage, some peak points might be merged as one point or some might be identified as false peaks.

Accordingly, a fuzzy rule based algorithm is developed to perform the following actions:

1. Local Filtering: filtering the false maximum and minimum peaks and pick up real peaks in each slice.

2. Global Combination: Merging some close points as one point and removing false points based on the global knowledge of the whole artifact rather than only a slice.

3. Edge Detection: locating corresponding points in different slices which belong to a specific yarn boarder. 
For this algorithm to work effectively, the maximum peaks of the autocorrelation function should be also calculated. It is expected that a max peak should be seen between two sequential minimum peaks which presents somewhere on top of the yarn where the light is maximum due to yarn convexity.

Figure 3 demonstrates the Global Combination mechanism. The distance is described by a distance fuzzy variable and merging of the points is carried out by the algorithm.

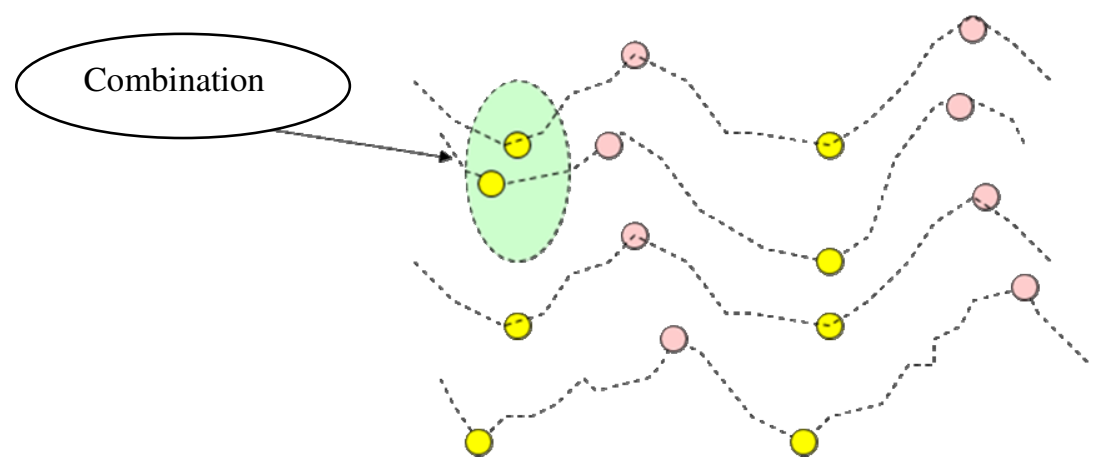

Fig. 3. Global combination mechanism

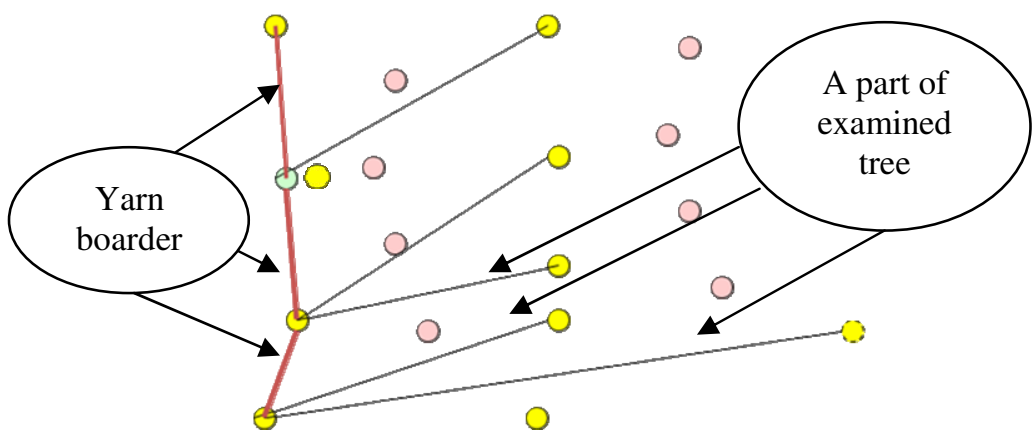

Fig. 4. Edge detection mechanism

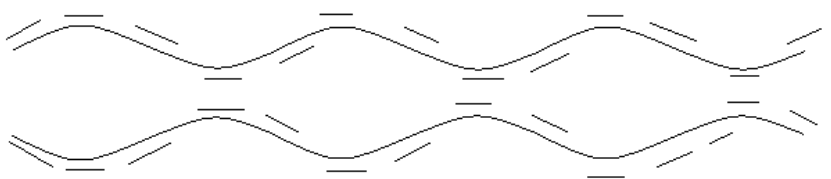

Fig. 5. Gradient pattern for yarn boarder 
Figure 4 shows the edge detection mechanism which locates corresponding points in different slices belonging to a specific yarn boarder. This mechanism searches for the points as a backtracking algorithm in a tree structure. It starts from the bottom left minimum peak in the artifact and finds different paths of closest points based on their fuzzy distance. The mechanism continues by considering next points recursively. This process creates a virtual tree. The fuzzy algorithm selects a path in the tree from root to leaves with the best estimation as a yarn boarder. The selected points are removed from the feature point set and the mechanism continues from next bottom left point. The algorithm ensures that a maximum peak point curve is located between two neighboring yarn boarders.

The yarn boarder gradient pattern is known and could be used for predicting the yarn edge path in tree structure (Figure 4). This gradient prediction is illustrated in Figure 5. The short lines indicate the gradients of the edge curve. The narrow cross sections show the locations where weft and warp are floating over each other. The shape is changing due to friction forces and compression in these areas. Finding the next point in the path has to satisfy the gradient pattern and the fuzzy rules.

The distance fuzzy variable membership function is shown in Figure 6. The Fuzzification of the distance is carried out by calculating the distance of point $\mathrm{p}$ relative to all the points in slice $i+1$ and normalizing the distances between 0 to 1 . The fuzzy set consists of very small, small, small-medium, large-medium, large and very large values.

In the final stage of the modeling, a quadratic spline interpolation is fitted to the yarn edge points for each identified yarn in weft and warp direction.

After tuning the rules, fuzzication \& defuzzication, and parameters estimation, the system is validated for 21 samples and has proved to be quite effective. Manual comparison has shown around $85 \%$ accuracy for the model. The model errors are found in artifacts with patterns or complicated woven structures.

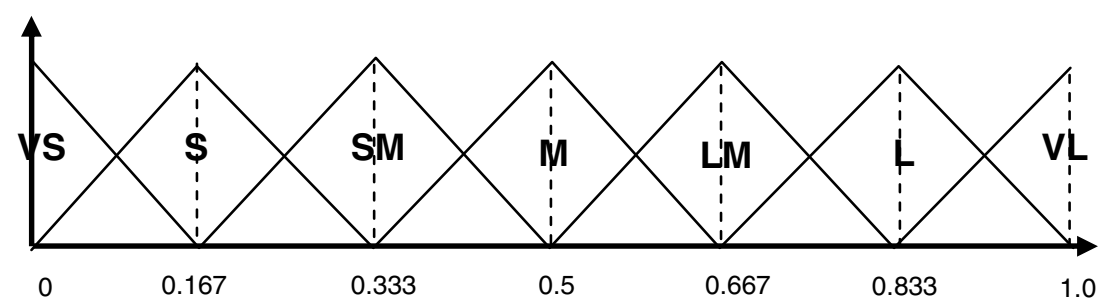

Fig. 6. The distance fuzzy variable membership function using for Fuzzification

\subsection{Crossed-States Detection}

A variety of methods have been suggested in the literature for crossed-states detection including employing texture orientation features in each one of the detected cells [11], normalized aspect ratio of an ellipse-shaped image at crossed points of the fabric [12], fuzzy c-means clustering [9, 10], and Fourier image analysis techniques [5, 7, 13-15]. The outcome of this stage is a weave pattern diagram showing the warp over weft or weft over warp in each cell of cross points. 


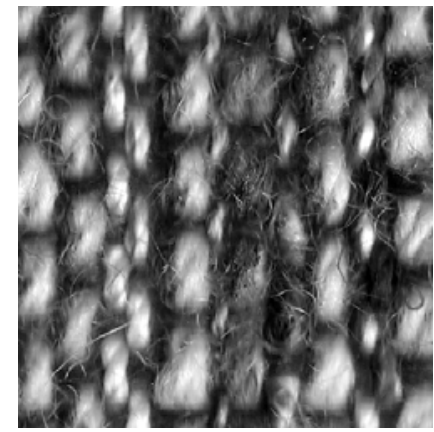

a.1

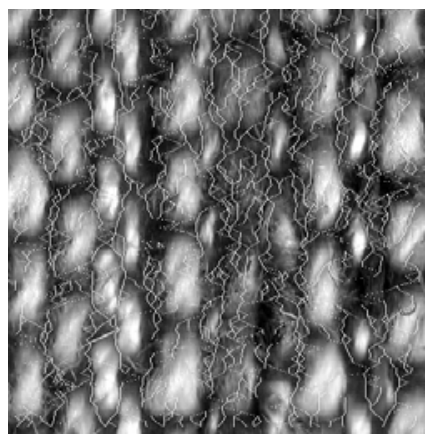

a. 2

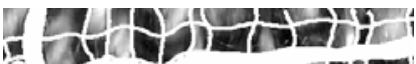

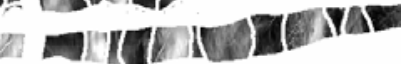

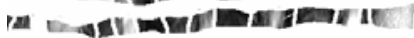

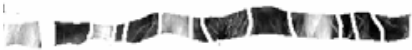

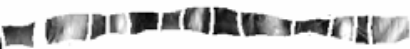

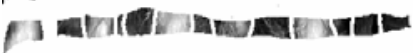

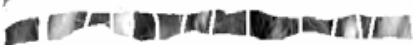

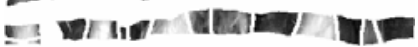

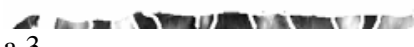
a.3

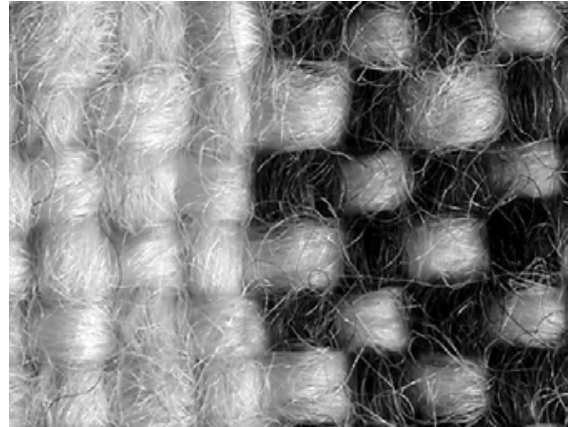

b. 1

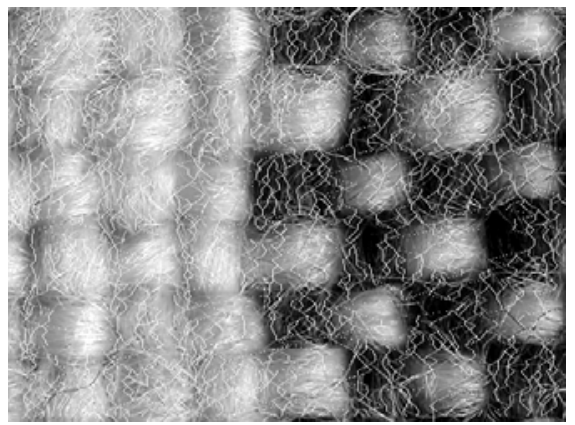

b. 2

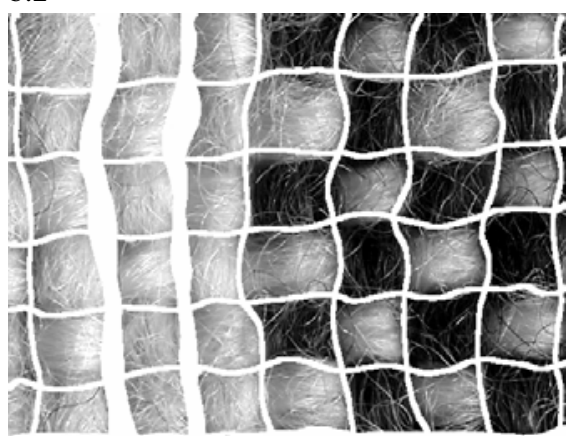

b. 3

Fig. 7. Crossed-points detection for two samples a and b

In this study, the yarn edges-texture orientation features of each side of the cell are calculated for each detected cell. The derived features are then transformed to frequency domain by deploying Fast Fourier Transform. The Frequency spectrum is finally analyzed for crossed-states detection process. This method is novel and has not been explored in textile industry for this problem.

Figure 7 illustrates the image processing steps applied to two samples of hand woven textiles of (a) and (b). Indexes 1 to 3 represent the following stages:

- Index 1: The original scanned images of textiles.

- Index 2: Textile image with its vertical and horizontal feature points.

- Index 3: Fuzzy rules implication for finding the yarn edges. 
A typical weave pattern diagram produced in crossed-states detection process is shown in Figure 8. Black cells are warp float areas and white ones are weft float areas.

The proposed method is verified by applying it to 21 artifact samples. The texture orientation method in frequency domain is evaluated with $87 \%$ accuracy compared with conventional methods with less than $70 \%$ accuracy.

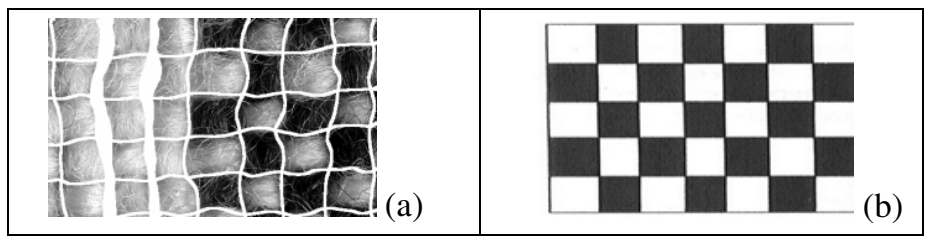

Fig. 8. Weave pattern diagram produced in crossed state detection

\section{Textile 3D Geometric Model Generation}

The textile structure and the measurements made of the weft and warp in pattern recognition process are deployed in developing the 3D geometric model of the fabric. The weft or warp yarn flow is modeled in two sections:

- The sinusoidal section that is used in transition flow when a weft or a wrap flows over or below the corresponding yarn

- The linear section that is used in constant flow

The weft and warp yarn cross-section is modeled as an ellipsoid with its dimensions set according to the pattern identified in the textile. Variation in the shape of the ellipsoid occurs due to internal, friction and compression forces between warp and weft yarns of the textile.

Figure 9 illustrates the linear flow section, sinusoidal flow section and yarn cross sections in both wrap and weft directions.

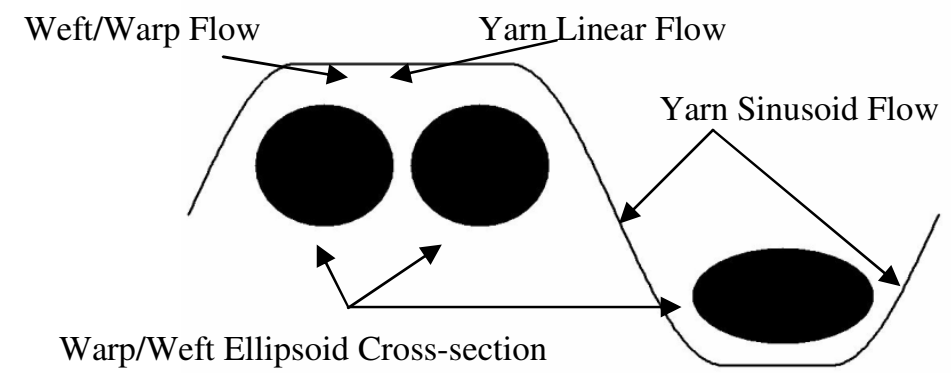

Fig. 9. Weft/Wrap flow in textile 3D geometric model 
Defining weft and warp yarn diameters by $\mathrm{d}_{\text {weft }}$ and $\mathrm{d}_{\text {warp }}$, respectively, the amplitude of weft and warp sinusoidal flow, $A_{\text {weft }}$ and $A_{\text {warp }}$ should satisfy (7):

$$
A_{\text {weft }}+A_{\text {warp }}=\frac{d_{\text {weft }}+d_{\text {warp }}}{2}
$$

$\mathrm{A}_{\text {weft }} / \mathrm{A}_{\text {warp }}$ ratio is dependent on the weft and wrap yarns raw material, composition and dimension. It determines the tension and compressibility of yarn and can be estimated empirically.

The weft and warp sinusoidal flow curve in 3D Cartesian coordinate space could be formulated as follow:

$$
\begin{aligned}
& z= \pm A_{\text {weft }} \operatorname{Sin}\left(\frac{2 \Pi x}{d_{\text {weft }}+d_{\text {warp }}}\right) \\
& z=\mp A_{\text {warp }} \operatorname{Sin}\left(\frac{2 \Pi y}{d_{\text {weft }}+d_{\text {warp }}}\right)
\end{aligned}
$$

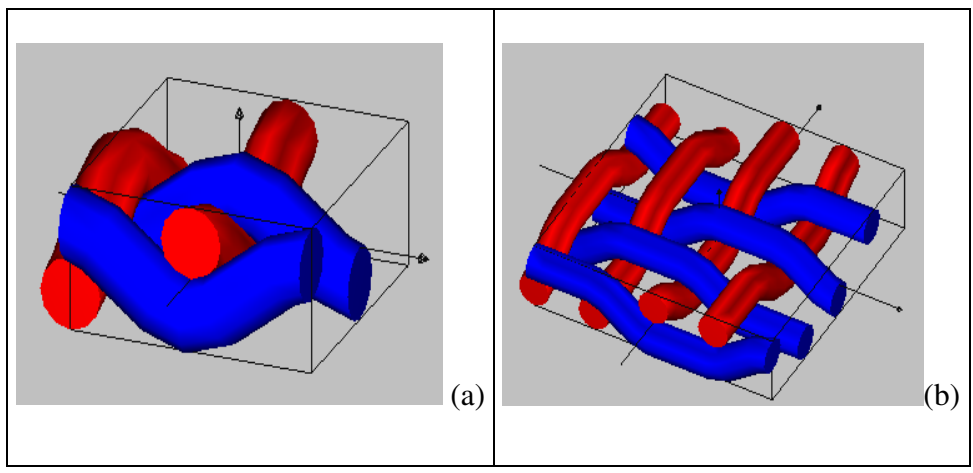

Fig. 10. 3D geometric model for: a) A plain artifact sample b) A twill artifact sample

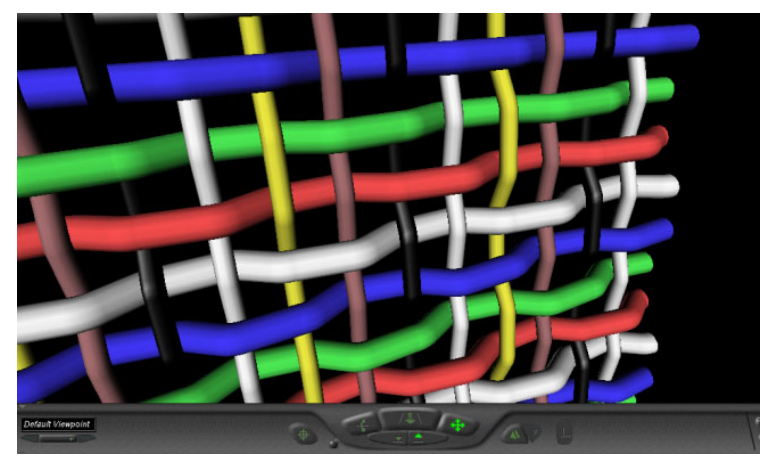

Fig. 11. An artifact VRML 3D geometric model in Virtual Reality Viewer 
The flow of the center of weft and warp yarns in $\mathrm{x}$ and $\mathrm{y}$ directions are shown by (8) and (9). By adding the ellipsoid cross-section for each specific point in curves, the textile $3 \mathrm{D}$ geometric model is generated. This model is converted to VRML by a developed automatic VRML generator. The VRML geometric model is displayed as a virtual reality world that can be explored by user.

Figure 10 demonstrates the 3D geometric model for the two artifacts samples. Figure 11 shows an artifact VRML 3D geometric model in the virtual reality viewer. The textile texture also could be added to the model for a realistic representation of the sample in the virtual reality world.

\section{Textile Mechanical Model Generation}

The Fabric Hand of a cloth or garment is defined as the overall fabric quality perceived through operations such as touching, squeezing, or rubbing [16]. Many factors affect the Fabric Hand including flexibility, compressibility, elasticity, resilience, density, surface contour (roughness, smoothness), surface friction and thermal characteristics of the fabric. The properties of the textile raw material, yarn structure, planner structure (woven or knitted pattern) and finishing treatment could be employed in a mechanical model for predicting the fabric hand and its factors including textile mechanical properties [17]. In many industrial applications, empirical modeling methods employing Kawabata machine are being deployed for fabric hand assessment rather than analytical or empirical models for estimating the textile physical and mechanical properties.

In this work, the mechanical properties of the textiles are estimated via an artificial neural network. In this stage of the work, the mechanical properties, which play a significant role in tactile perception of the hand-woven textile, are calculated. This model is faster and cheaper than textile empirical models and could be easily deployed in haptic modeling of textiles.

The neural network system developed in this study to recognize the textile physical properties is a three layer Perceptron. The unsupervised back-propagation learning method is used for tuning the system weights for any specific problem. The first layer has 16 neurons for 16 inputs. The output layer has 3 neurons for 3 outputs. The hidden layer size is different for any specific problem. There are different ways available to estimate the size of the hidden layer. One of the famous empirical rules is the mean of input and output layer size. In this approach, different hidden layer sizes from 5 to 15 neurons are examined to find the minimum error in back-propagation training. The average of input and output layer size is 10 and hence a boundary around this point is selected. The hidden layer, learning ratio and primitive weights are estimated based on the training data. The system inputs include yarn raw materials, weft and warp dimensions and weft and warp structure. The outputs of the system include static friction, dynamic friction and compressibility.

In the training system, the raw materials are limited to natural materials - wool, linen, cotton and silk. The yarn dimension expressing the average diameter and the yarn structure is limited to yarn toughness indicator that encapsulates the yarn structure parameters and yarn degree of twist. Figure 12 demonstrates the system architecture for a specific weave pattern. 

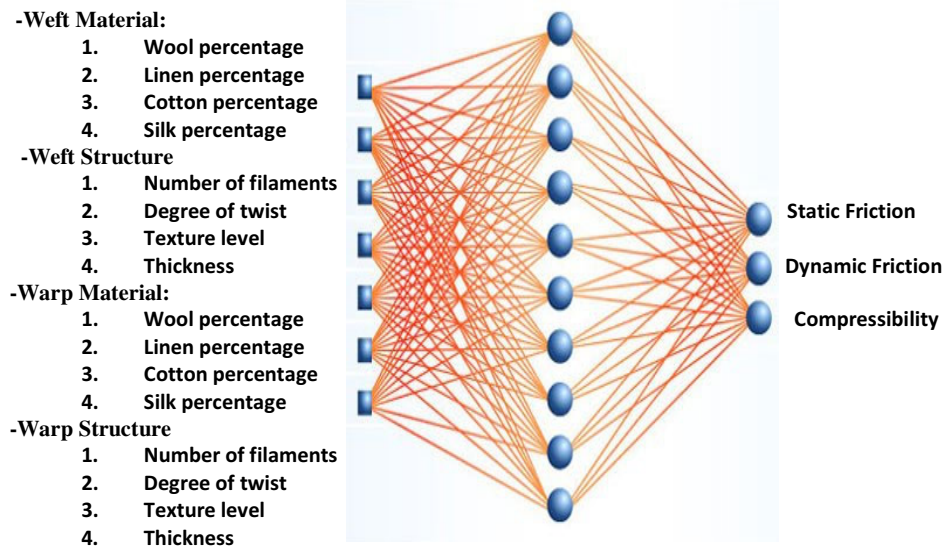

Fig. 12. Neural Network system for textile mechanical \& physical properties prediction

\section{Haptic Model Generation}

The haptic model is generated by superimposing the textile mechanical model over 3D geometric model. The implementation is achieved through Reachin computing platform. The Reachin API is a multi-sensory rendering engine that integrates visual and haptic rendering through the use of one single scene graph. It parses the graph and provides the 3D presentation of an object as well as providing tactile perception for the world. It is system and haptic device independent which makes it suitable for future applications with other haptic devices. Reachin API is based on the concept of the scene graph. A scene graph is a hierarchical data structure that describes a 3D scene. It holds the geometry of all objects in the scene and their relative positions, appearance attributes such as color, transparency, textures and surfaces and also light sources, viewing position and information about the scene. A scene graph provides a framework for managing objects in a scene, and makes it easy to express the relationship between those objects [18].

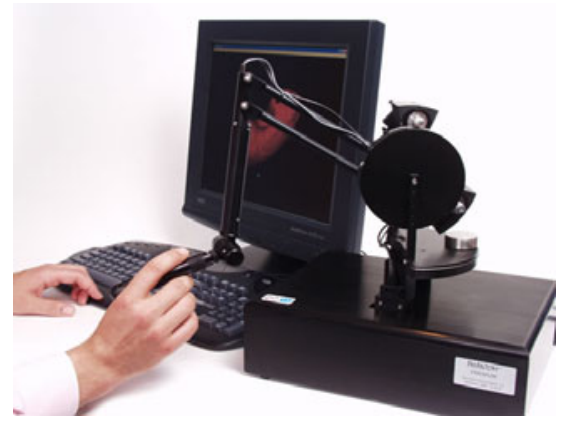

Fig. 13. The 6 DOF Phantom haptic device 
Several hand-woven artifacts have been modeled with the proposed methodology. These models have been rendered in Virtual Manipulation Laboratory at University of Wollongong and the tactile interaction has been achieved through a 6 DOF Phantom haptic device. Figure 13 illustrates the 6 DOF Phantom device which has been employed in the laboratory for tactile interaction.

\section{Validation}

The 3D geometric model has been generated for 21 samples. From them, 13 twill and plain samples have been selected for mechanical/physical model generation. The haptic model generation method developed in this work is applied to all the samples. In addition, other haptic models using Bumpmapsurface and FrictionImageSurface from Reachin have been generated for comparison and validation. Several experiments were designed and implemented to examine the model validity and performance of the methodology.

In one of the experiments, 5 observers were selected. Each one was instructed to touch the 13 hand-woven artifact samples and the haptic models developed for them by using the Phantom haptic device. The observers were given 3-5 minutes to examine each real artifact and the corresponding haptic model. For accurate comparison, a pen with the same shape as haptic device pen was given to each candidate. The observers were asked to touch the real artifact with the pen and the haptic model with Phantom device. They would then give a score of 0 to 10 for each sample, with 0 indicating no similarity and 10 meaning complete similarity. The candidates were told to pause for 2-3 minutes between samples. The experiment took about 1.5 to 2 hours for each observer. They were then asked to repeat the experiment for Bumpmapsurface and FrictionImageSurface methods in the following day. The sequence of samples was changed in each set of experiments, eliminating the previous judgment on the result.

The average of similarity scorers gathered from five candidates for 13 samples are provided in Table 1 for each method.

Table 1. The subjective experiment result for 13 samples and 3 different methods

\begin{tabular}{|c|c|c|c|c|c|c|c|c|c|c|c|c|c|c|}
\hline Sample number & $\mathbf{1}$ & $\mathbf{2}$ & $\mathbf{3}$ & $\mathbf{4}$ & $\mathbf{5}$ & $\mathbf{6}$ & $\mathbf{7}$ & $\mathbf{8}$ & $\mathbf{9}$ & $\mathbf{1 0}$ & $\mathbf{1 1}$ & $\mathbf{1 2}$ & $\mathbf{1 3}$ & $\begin{array}{c}\text { The Method } \\
\text { Average }\end{array}$ \\
\hline FrictionImageSurface & 3.6 & 4.2 & 2.6 & 6 & 5.2 & 4.8 & 6.2 & 4.4 & 3.2 & 3.6 & 4.2 & 5.4 & 6.4 & $\mathbf{4 . 6}$ \\
\hline BumpmapSurface & 4.2 & 3.8 & 3.4 & 5.2 & 6.4 & 5.6 & 5.8 & 4.2 & 5.6 & 3.2 & 5.8 & 7.4 & 6.8 & $\mathbf{5 . 2}$ \\
\hline Proposed method & 4.4 & 7.2 & 6.8 & 8.2 & 7 & 8.8 & 7.8 & 6.6 & 7.8 & 6.8 & 8.4 & 8.8 & 7.2 & $\mathbf{7 . 4}$ \\
\hline
\end{tabular}

As shown in the table, the subjective assessment of the three methods has identified the highest similarity score for the method proposed in this work using the developed 3D geometric and physical models. The FrictionImageSurface method is the worst one. The proposed method shows an average similarity score of $74 \%$ which is about $60 \%$ lead over FrictionImageSurface method and around $40 \%$ improvements over BumpmapSurface. 
In the second experiment, each observer was exposed to 3 artifact samples and the haptic model of one of the samples. The model was randomly chosen and the observers were not informed of the corresponding sample. The observers were then expected to identify the sample by examining both the model and the samples. Each candidate was given three minutes for each model. The experiment was repeated for FrictionImageSurface and BumpmapSurface haptic models as well as the model developed in this work. Fifteen haptic models were observed by each candidate, 5 in each group. Therefore 25 tests were carried out for each haptic group.

The overall results are provided in Table 2, illustrating the number and percentage of true identification for each haptic model.

Table 2. Number of True identification in 25 tests for each haptic group

\begin{tabular}{|c|c|c|}
\hline & $\begin{array}{c}\text { Number of True } \\
\text { Identification (25 total ) }\end{array}$ & True Identification Percentage \\
\hline FrictionImageSurface & 12 & $48 \%$ \\
\hline BumpmapSurface & 16 & $64 \%$ \\
\hline Our method & 21 & $84 \%$ \\
\hline
\end{tabular}

As highlighted in the table, identification based on the proposed method has again score the highest with true identification percentage of $84 \%$. The true identification rates for FrictionImageSurface and BumpmapSurface are only $48 \%$ and $64 \%$ respectively. The result shows almost $75 \%$ and $31 \%$ improvements over FrictionImageSurface and BumpmapSurface respectively.

Both experiments prove the validity and efficiency of the proposed model with acceptable success rates.

\section{Conclusion}

The development of a generic approach for 3D haptic-rendered modeling of handwoven textile artifacts was reported in this paper. The proposed methodology relies on processing of the still images of the artifact using computational intelligence as well as the knowledge of the artifact material and yarn structure. Effort was made to ensure that the developed mechanism was a faster and more reliable alternative for textile modeling compared to the costly and time consuming manual fabric hand assessment. The approach was also designed to build the haptic model of the textile based on an accurate 3D geometric model of the artifact rather than conventional 2D mesh structure used for graphical representation.

Future work will concentrate on pattern recognition and neural network enhancement as well as customizing the physical simulator and haptic render engine for handwoven artifacts. 


\section{References}

1. Govindaraj, M., et al.: Haptic simulation of fabric hand. Technical report, National Textile Research Annual Report and in Eurohaptics 2003 Conference (2003)

2. Mäkinen, M., et al.: Influence of Physical Parameters on Fabric Hand. In: Proceedings of the HAPTEX 2005 Workshop on Haptic and Tactile Perception of Deformable Objects, Hanover (2005)

3. Magnenat-Thalmann, N., Bonanni, U.: Haptics in virtual reality and multimedia. IEEE Multimedia 13(3), 6-11 (2006)

4. Shidanshidi, H., et al.: 3D geometric modelling of hand-woven textile. SPIE, San Jose (2008)

5. Xu, B.G.: Identifying fabric structures with Fast Fourier Transform techniques. Textile Research Journal 66(8), 496-506 (1996)

6. Lachkar, A., Gadi, T., Benslimane, R., D’Orazio, L.: Textile Woven Fabric Recognition using Fourier Image Analysis Techniques: Part I: A Fully Automatic Approach for Crossed-points Detection. J. Text. Inst. 94(1), 194-201 (2003)

7. Ravandi, S.A.H., Toriumi, K.: Fourier-Transform Analysis of Plain Weave Fabric Appearance. Textile Research Journal 65(11), 676-683 (1995)

8. Jeon, B.S., Bae, J.H., Suh, M.W.: Automatic recognition of woven fabric patterns by an artificial neural network. Textile Research Journal 73(7), 645-650 (2003)

9. Kuo, C.F.J., Shih, C.Y., Lee, J.Y.: Automatic recognition of fabric weave patterns by a fuzzy C-means clustering method. Textile Research Journal 74(2), 107-111 (2004)

10. Kuo, C.F.J., Shih, C.Y., Lee, J.Y.: Repeat pattern segmentation of printed fabrics by hough transform method. Textile Research Journal 75(11), 779-783 (2005)

11. Lachkar, A., Benslimane, R., D’Orazio, L., Martuscelli, E.: Textile Woven Fabric Recognition using Fourier Image Analysis Techniques: Part II - texture analysis for crossedstates detection. J. Text. Inst. 96(3), 179-183 (2005)

12. Kang, T.J., Kim, C.H., Oh, K.W.: Automatic recognition of fabric weave patterns by digital image analysis. Textile Research Journal 69(2), 77-83 (1999)

13. Campbell, J.G., Murtagh, F.: Automatic visual inspection of woven textiles using a twostage defect detector. Optical Engineering 37(9), 2536-2542 (1998)

14. Kuo, C.F.J., Tsai, C.C.: Automatic recognition of fabric nature by using the approach of texture analysis. Textile Research Journal 76(5), 375-382 (2006)

15. Rallo, M., Escofet, J., Millan, M.S.: Weave-repeat identification by structural analysis of fabric images. Applied Optics 42(17), 3361-3372 (2003)

16. Hui, C.L.: Neural Network Prediction of Human Psychological Perceptions of Fabric Hand. Textile Research Journal (May 2004)

17. Hatch, L.K.: Textile Science. West Publishing Company, Minneapolis (1993)

18. AB, R.T., Reachin API 3.2 Programmer's Guide (1998-2003) 